\title{
Linking Clinical Events in Elderly to In-home Monitoring Sensor Data: A Brief Review and a Pilot Study on Predicting Pulse Pressure
}

\author{
Mihail Popescu and Elena Florea \\ Health Management and Informatics Department, \\ University of Missouri, Columbia, Missouri, USA \\ popescum@missouri.edu; evfc99@mizzou.edu
}

Technology has had a tremendous impact on our daily lives. Recently, technology and its impact on aging has become an expanding field of inquiry. A major reason for this interest is that the use of technology can help older people who experience deteriorating health to live independently. In this paper we give a brief review of the in-home monitoring technologies for the elderly. In the pilot study, we analyze the possibility of employing the data generated by a continuous, unobtrusive nursing home monitoring system for predicting elevated (abnormal) pulse pressure ( $\mathrm{PP}$ ) in elderly ( $\mathrm{PP}=$ systolic blood pressure-diastolic blood pressure). Our sensor data capture external information (behavioral) about the resident that is subsequently reflected in the predicted PP. By continuously predicting the possibility of elevated pulse pressure we may alert the nursing staff when some predefined threshold is exceeded. This approach may provide additional blood pressure monitoring for the elderly persons susceptible to blood pressure variations during the time between two nursing visits. We conducted a retrospective pilot study on two residents of the TigerPlace aging in place facility with age over 70 , that had blood pressure measured between 100 and 300 times during a period of two years. The pilot study suggested that abnormal pulse pressure can be reasonably well estimated (an area under ROC curve of about 0.75 ) using apartment bed and motion sensors.

\section{INTRODUCTION}

TigerPlace [Rantz et al. 2005] is an independent living facility for seniors designed and developed as a result of collaboration between Sinclair School of Nursing, Columbia, Missouri and Americare Systems Inc. of Sikeston, Missouri. Senior citizens move to TigerPlace in one of the 31 apartments, and do not need to move again regardless of any change in their care needs. TigerPlace features care provided for

Copyright(c)2008 by The Korean Institute of Information Scientists and Engineers (KIISE). Permission to make digital or hard copies of part or all of this work for personal or classroom use is granted without fee provided that copies are not made or distributed for profit or commercial advantage and that copies bear this notice and the full citation on the first page. Permission to post author-prepared versions of the work on author's personal web pages or on the noncommercial servers of their employer is granted without fee provided that the KIISE citation and notice of the copyright are included. Copyrights for components of this work owned by authors other than KIISE must be honored. Abstracting with credit is permitted. To copy otherwise, to republish, to post on servers, or to redistribute to lists, requires an explicit prior permission and/or a fee. Request permission to republish from: JCSE Editorial Office, KIISE. FAX +82 25211352 or email office@kiise.org. The Office must receive a signed hard copy of the Copyright form. 
the seniors by Senior Care, a licensed home health agency owned by the Sinclair School of Nursing, providing on-going assessment, early illness recognition, and health promotion activities. An outstanding building, designed to accommodate the needs of seniors and their pets makes TigerPlace an attractive place to live.

A primary goal of TigerPlace is to help the residents not only manage their illnesses but also stay as healthy and independent as possible. The use of sensor technology to provide early identification of problems in mobility and cognition is currently being implemented. The integrated monitoring system under development has three main components: (a) the in-home monitoring system, (b) an event-driven, video sensor network that hides identifying features of the residents, and (c) a reasoning component that fuses sensor and video data and analyzes patterns of behavioral activity. Each residence included in the network has a $\mathrm{PC}$ that is connected to a main server through a wired network connection. Data is monitored and collected at the main server for research purposes. The data manager collects data from the sensors of the In-home monitoring system, date-time stamps the data, and logs it into a file that is sent to the secure server.

The in-home monitoring system consists of several types of sensors mounted in different places, throughout the residents' apartments. They are motion sensors, bed sensors, and temperature sensor. The motion sensors are placed in various places, such as bathroom, bedroom, kitchen, living room, etc. and some of the residents have this type of sensors installed on the door of the refrigerator, kitchen cabinets and even drawers. They are capable to capture resident motion through his/her apartment by emitting a signal (firing) as often as there is movement around them. The bed sensors are in fact sets of sensors, composed of a sensor strip across the bed and a motion sensor attached to the bed headboard. The sensor strip is able to keep track of the resident's movement in the bed, namely restlessness, heart rate and breathing, as long as the resident is on the bed. The motion sensor attached to the headboard captures motion around the bed - when the resident gets in and out of the bed. The sensor strip and motion sensor attached to the bed are connected together and they function similarly to the motion sensors mention previously: they fire as long as they detect activity around them. As stated before, unlike the motion sensors, the sensor strip captures three types of activities, which are structured on three or four levels of severity as follows:

- Four levels of restlessness: low, high, higher, highest depending on the duration of movement.

- Three levels of breathing: low, normal, fast depending on the number of breaths per minute.

- Three levels of heart rate: low, normal, fast depending on the number of heart beats per minute.

\section{STATEMENT OF THE PROBLEM}

The aim of this study is to find the connection between the change in health status and sensor data produced by the in-home monitoring system. The primary goal is to find a link between abnormal levels of daily activities and vital signs of residents using data mining algorithms. An abnormal sleep pattern and/or low level of motion 
may be related to an elevated blood pressure or to a heart attack. Our sensor data capture external information (behavioral) about the resident that is subsequently reflected in the predicted PP. By continuously computing the pulse pressure and comparing it with the measured trend we may alert the nursing staff when some predefined variability limits are exceeded. This approach may provide additional blood pressure monitoring for the elderly persons susceptible to blood pressure variations during the time between two nursing visits. In addition, the comparison of the computed and measured pulse pressure trends over longer periods of time may provide additional warnings of abnormal unreported clinical events. The algorithms will be validated using the clinical data of the participants connected to the in-home monitoring system.

\section{RELATED WORK}

\subsection{Issues with Aging Population}

The proportion of elderly in the population is growing at a rapid rate in countries around the world. Many of these seniors prefer to live independently for as long as they are able, despite the onset of conditions such as frailty and dementia [Cuddihy et al. 2007]. It is well known that the major cause for placement of elderly people in an institution is loss of autonomy due to physical or cognitive impairment [Chan et al. 2005]. With such a high and continued increasing average life expectancy rate, followed by the dramatic change in trend of the proportion of elderly in the total population, medical care for senior citizens, age 60 and over, is becoming progressively more important. Solutions are needed to enable independent living while enhancing seniors' safety and their families' peace of mind [Cuddihy et al. 2007; Rowan and Mynatt 2005].

Aging adults are often stereotyped as purposefully masking any decline in abilities to avoid outside intervention and this fact leads to the concern held by adult children about their aging parents: knowing if there are subtle declines in capabilities or behavior of their parents [Rowan and Mynatt 2005]. Elderly patients are particularly at-risk for late assessment of cognitive changes due to many factors: their impression that such changes are simply a normal part of aging, their reluctance to admit to a problem, their fear of being institutionalized and even the failure of physicians to fully assess their cognitive function due to the belief that no intervention is possible [Hayes et al. 2004]. Even ongoing or post-treatment monitoring of patients through periodic but infrequent office visits has many limitations. Relying on self-report by the patient or their family is also unreliable. Current clinical monitoring approaches may miss important fluctuations in behavior and health state [Hayes et al. 2004]. This problem still remains in nursing homes. Physicians might visit their patients for only a short period of time, usually once a week. Assessment of a patient's progress is thus based mainly on reports from staff (nurses and nurse assistants). The reports may be incomplete, or even biased due to schedule shift and the fact that each staff person has to care for many patients [Chen et al. 2007]. This may result in insufficient observation for monitoring either progressive change, or brief and infrequent occurrences of aberrant activity that might lead to diagnosis of some diseases. 


\subsection{Pulse Pressure - Predictor of Multiple Diseases}

Hypertension and its treatment are of particular relevance to the elderly and very elderly [Peters et al. 2007]. As systolic blood pressure (BP) tends to rise with age, at least in most populations, relatively high percentages of elderly population are classified as hypertensive. More specifically, it is systolic BP that rises more linearly with age, although it may plateau slightly at approximately 80 years, whereas diastolic BP rises until approximately 60 years and falls thereafter [Peters et al. 2007]. This is of particular interest when it is remembered that those aged over 80 years are among the fastest growing sector of the population, and are likely to be hypertensive and to have an isolated systolic hypertension with larger pulse pressure (PP) values (the difference between systolic BP and diastolic BP).

The majority of individuals older than 70 years have a widened pulse pressure resulting from age-related stiffening of the central elastic arteries and systolic hypertension. A widened pulse pressure is associated with cardiovascular risk factors such as diabetes, hypertension, and smoking. It also predicts a higher risk of subsequent cardiovascular events [Peters et al. 2007; Blacher et al. 2000], coronary heart disease [Franklin et a]. 2001], renal disease, heart failure [Swaminathan and Alexander 2006], and mortality [Safar et al. 2004; Mitchell et al. 2007; Glynn et al. 2000; Lee et al. 1999], particularly in the elderly. According to Safar et al. [2004], PP of $60 \mathrm{~mm} \mathrm{Hg}$ is a strong mechanical factor predicting cardiovascular mortality. Based on epidemiological studies, it is well accepted that PP above the critical level of $60 \mathrm{~mm} \mathrm{Hg}$, causes particular risk in patients. Such cutting point, has been established for PP on the basis of epidemiological studies indicating the lower level of PP at which renal, cerebral, and most ischemic cardiopathies (myocardial infarctions) occur [Safar et al. 2004]. In contrast, according to Peters et al. [2007] and Swaminathan and Alexander [2006], no practical cut-off value exists for differentiating normal pulse pressure from abnormal pulse pressure. Swaminathan and Alexander [2006] stated that most patients with systolic hypertension have elevated PP (>60) and by the age of 75 , the majority of individuals have elevated PP. In addition, PP seems to

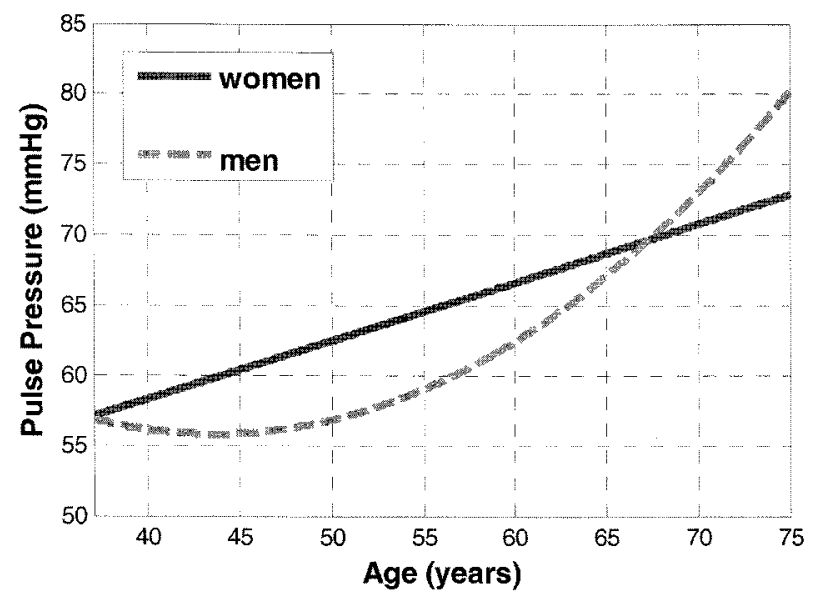

Figure 1. PP increase with age in men and women (from Safar et al. 2004). 
increase with age (Figure 1) [Safar et al. 2004] and for any given age, men have a 5\%$10 \%$ higher PP than women [Swaminathan and Alexander 2006]. In Figure 1 we used the following regression equations: $y=0.42 x+42$ for women and $y=0.025 x^{2}$ $+2.15 x+102$ for men, calculated based on data from [Safar et al. 2004].

\subsection{Sensor Technology used in Previous Research}

With the maturity of sensing and pervasive computing techniques, extensive research is being carried out in using sensor networks for home care environments. Homebased healthcare encourages the maintenance of physical fitness, social activity and cognitive engagement helping the elderly to function independently in their own homes [Yang et al. 2004]. Health monitoring in home environments can be accomplished by 1) ambulatory monitors that utilize wearable sensors and devices to record physiological signals; 2) sensors embedded in the home environment and furnishings to unobtrusively collect behavioral and physiological data; or 3) a combination of the two [Alwan et al. 2006].

Sensors in the first category include accelerometers [Davis and Fox 2007], cell phones [Eagle and Pentland 2006], and wireless body area network (WBAN) that monitor the user's heart rate and locomotive activity [Otto et al. 2006]. Sensors in the second category include field sensors [Hayes et al. 2004], contact sensors [Hayes et al. 2004; Gil et al. 2007; Cuddihy et al. 2007], motion, location or infrared sensors [Hayes et al. 2004; Chan, Campo, and Esteve 2005; Shieh et al. 2006; Gil et al. 2007; Virone et al. 2002; Alwan et al. 2006; Cuddihy et al. 2007], strain sensors [Rowan and Mynatt 2005], low power smart cameras [Williams et al. 2007], microphones [Fogarty et al. 2006], thermal imaging sensors [Sixsmith and Johnson 2004], switch sensors [Tapia et al. 2004], pressure sensors [Gil et al. 2007], and electrical sensors [Gil et al. 2007]. A study by Virone et al. [2006] is an example of health monitoring in home environments accomplished by using a combination of wearable devices and sensors embedded in the home environment. The authors of this study used unobtrusive area and environmental sensors deployed in the assisted living environment (rooms, hallways, units, furniture) including: motion, video camera, temperature, humidity, acoustic, smoke, dust, gas, etc. combined with wearable interactive devices equipped with a variety of sensors (such as heart-rate, heartrhythm, temperature, oximeter, accelerometer). Similarly, Teaw et al. [2005] combined wireless sensor networks, existing RFID (Radio Frequency Identification) and Vital Sign Monitoring technology to simultaneously monitor vital signs while keeping track of the users' location.

Many researchers have explored wearable sensors for their advantages in the area of continuous monitoring. They provide more precise tracking methodologies as long as the subject wears the devices at all times [Hayes et al. 2004]. Accelerometers offer a feasible tool for the assessment of several aspects of PA in older people. They provide valuable data for the assessment of volumes, intensity and patterns of activity (PA) across the daily and weekly patterns [Davis and Fox 2007]. Eagle and Pentland [2006] used cell phones as wearable devices for their ability to measure information access and use in different contexts, recognize social patterns in daily 
user activity, infer relationships, and identify socially significant locations.

Sensors embedded in the home environment and furnishing proved to have many advantages in the area of health monitoring. They are non-intrusive and easily accepted by elders in their residences [Alwan et al. 2006; Rowan and Mynatt 2005], inexpensive [Shieh et al. 2006], do not require the occupant to push any buttons or wear any devices [Rowan and Mynatt 2005], and are familiar fixtures in home security systems [Cuddihy et al. 2007]. Wireless sensors provide flexibility of installation of additional sensors in a very short time and minimally disruptive to the subject [Hayes et al. 2004]. Non contact sensors are considered more practical than wearable sensors. For example, a switch sensor in the bed can strongly suggest sleeping, and pressure mat sensors can be used for tracking the movement and position of people [Tapia et al. 2004]. Also, data provided by switches and motion sensors is reliable and very easy to process [Chen et al. 2007] while strain sensors provide relatively clean and reliable signals [Rowan and Mynatt 2005]. Generally, wireless sensors have a low power consumption and low production cost, can be embedded in different places or objects at home or they can become wearable by integrating them into clothing or small apparel items like watches or jewelry [Shieh et al. 2006]. Particularly, thermal imaging sensors have the advantage that sensing of a subject's motion can occur locally, within the detector, using only a modest processor [Sixsmith and Johnson 2004]. This is due to the relative ease of interpretation of data generated by the sensor. They are cost-effective, the low-level data lacks detail and there is no need to transmit data outside the detector. The system composed of thermal imaging sensors seems less intrusive to users and they generally view thermal imaging more positively than cameras [Sixsmith and Johnson 2004]. A sensor of this type can reliably locate and track a thermal target in the sensor's field of view, providing size, location, and velocity information.

Assistive technology should be very carefully designed because it may be rejected if it detracts from the aesthetics of the home, leads an elder to feel spied upon, or creates a feeling of embarrassment over the need for assistance [Hayes et al. 2004]. Hayes et al. [2004] identified many negative reactions to the intrusion of sensors into the living space, including objections to the potential for damage caused by the adhesive used for installation, concern that sensors were placed in locations accessible by children or pets, and objections to the placement of cameras and microphones in the home. Elderly individuals are frequently unwilling to adapt even to small changes in their environment, including wearable sensors in their clothing [Chen et al. 2007]. Requiring the subject to wear a device at all times is obtrusive and can result in non-compliance [Hayes et al. 2004]. For example, among challenges posed by cell phones as wearable devices for continuous monitoring are human-induced errors by the phone being off or left on but separated from the user [Eagle and Pentland 2006].

Even simple, not wearable sensors, can impact behavior and have other disadvantages. One of the two participants in a study by Tapia et al. [2004], explained that being sensed did cause her to alter some behaviors as she always made sure to wash her hands after using the bathroom. As opposed to wearable sensors, the data provided by switches and motion sensors cannot provide detailed information. For example, a 
motion sensor can only tell that there is a person in the monitored area, but cannot tell the exact location [Chen et al. 2007]. In general, installation of the non wearable sensors is not always easy. Sensors requiring installation by the end users cause significant issues: end-users can make a variety of errors, often due to the directional requirements of sensors or uncertainty over exactly where a sensor needs to be positioned. Even if the sensors are not required to be installed by the end users, sometimes their installation creates real challenges. For example, installation of strain sensors requires access to the underside of the floor, making it impossible to use these sensors on the second floor of an existing home. This fact led to the idea of using wireless motion sensors in future deployable version of an awareness system instead of the strain sensors used in early versions [Rowan and Mynatt 2005].

More complex systems based on cameras or microphones are not challenge free. One of the major challenges of vision-based systems is the apparent intrusion of privacy because of the way the image data is transmitted and analyzed [Yang et al. 2004]. Existing systems are mainly based on central processing units where multiple video streams are received and processed. Due to the complexity of the computational tasks, data buffering and storage is often required, giving rise to major concerns over practicality and the potential intrusion of privacy [Yang et al. 2004]. Furthermore, Tapia et al. [2004] affirmed that in the case of complex sensors such as cameras or microphones, the recognition inference problem is often seriously underconstrained. Computer vision sensing, for example, often works in the laboratory but fails in real home settings due to clutter, variable lighting, and highly varied activities that take place in natural environments. Little of the work with video and audio processing in the lab has been extensively tested in the field [Tapia et al. 2004]. Perhaps just as importantly, because sensors such as microphones and cameras are so general and most commonly used as recording devices, they can be perceived as invasive and threatening by some people. The acclimation period to more invasive sensors such as cameras would be substantially longer. Some people would not agree to studies involving video observations or others would agree to those studies restricting cameras from the bathroom [Tapia et al. 2004].

The deployment of sensor networks in a home environment, however, requires careful consideration of user compliance and privacy issues. The choice of sensing technology that does not identify the person reduces the privacy concerns. Older adults are concerned about maintaining a careful balance between privacy and autonomy [Rowan and Mynatt 2005]. While both are important, aging often necessitates some compromise. Giving up some privacy in order to maintain autonomy is a valid choice. There is, however, a limit to how much and what type of sensor technology provides the correct balance [Rowan and Mynatt 2005]. Referring to awareness systems Romero et al. [2007], affirmed that achieving awareness is how to achieve a balance between what information people would like to know about others or, conversely, to make known to others. There is a tradeoff between trying to address the need to communicate and the need for privacy, which gets reflected in the level of detail/abstraction of information displayed [Romero et al. 2007].

Whatever the practical benefits might be, users might not accept the technology if they believe it impinges on their privacy and lifestyle. The ethical aspects of 
implementing such technology are also important. In particular, these technologies should be used only where end users or their caregivers understand the technology and can provide informed consent. According to Sixsmith and Johnson [2004] technology solutions should be one of a range of care options available to people. Ensuring that implementation does not lead to a technological "fix" for all the problems facing the elderly should be central to good practice.

It is essential to create end user acceptance of the assistive technology in their homes. One way is by providing the elderly a sense of control over Ambient Intelligence concepts and technologies [Ruyter and Pelgrim 2007]. In a case of an awareness system tested by Rowan and Mynatt [2005], the authors concluded that older adults accept a sort of sensing in their home and a visualization of that data for the adult child, and more importantly, they welcome and rely on the sensing or they circumvent and game the system. Another way to create user acceptance of the systems in their homes is to design systems that are unobtrusive and do not require the users to adjust their daily routine. To achieve this, the sensor nodes need to be small enough to be placed discreetly in appropriate locations. Ideally the sensor nodes would scavenge power from their surroundings, enabling an autonomous sensor network to be installed easily and to operate for extended periods of time with little or no outside intervention [Yang et al. 2004].

\subsection{Data Mining Algorithms used in Previous Research}

The use of data mining and on-line analytical processing (OLAP) is potentially interesting in this context of continuous monitoring because of the possibility of exploring, detecting and predicting changes in the level of activity of people's movement that may reflect change in well-being. The measure of activity, presence in locations and interaction with objects might provide information to assist understanding of patterns in people's behavior [Gil et al. 2007]. If there are regular patterns in the life of a person, changes to such patterns could suggest a change that should be followed up in a dialogue between a care provider and that person.

Most of the research in the area of continuous monitoring is related to identification of activities of daily living, which is an important predictor of cognitive and physical decline of the elders [Tapia et al. 2004; Atkinson et al. 2005]. For intelligent analyses the sensors are grouped together according to the domain knowledge and specific activities of interest. Thus each activity of daily living will have an associated group, which fuses data from all the sensors that are related to that activity. Yang et al. [2004] suggested that rather than gathering data from all sensors and carrying out an overall data-mining algorithm, a focused and more efficient algorithm can be applied to each of the activities according to the available knowledge and the data collected by the corresponding sensor group.

Usually three types of activities are included in the analysis phase: long-term trends, significant patterns, and associations among patterns [Yang et al. 2004]. The analysis of well-being is challenging, as abnormal patterns of behavior are difficult to identify. Additionally, what it is considered to be normal for one person can be abnormal for someone else. Moreover, people have a tendency to change the way 
they do things without necessarily being affected by deterioration in their physical or mental abilities. For example, the same person can behave in quite a different manner depending on the weather conditions. Therefore, interactive and adaptive algorithms are necessary to handle such analysis with the particularities of each individual in mind. One of the limitations of ambient sensing with simple sensors is that it is difficult to infer detailed changes in activity and those physiological changes related to the progression of disease. In fact, even for the detection of simple activities such as leaving and returning home, the analysis steps involved can be complex [Yang et al. 2004].

From the computational aspect, various data analysis techniques have been used including classification algorithms, as decision trees [Chen et al. 2007; Gil et al. 2007], SVM [Chen et al. 2007; Williams et al. 2007], logitboost [Chen et al. 2007], rule based approach [Alwan et al. 2006], mixture models as Gaussian mixture [Eagle and Pentland 2006], pattern recognition algorithms [Fogarty et al. 2006], sophisticated data mining techniques and machine learning algorithms like Markov chain [Eagle and Pentland 2006], artificial neural networks [Sixsmith and Johnson 2004], and Bayesian models [Tapia et al. 2004]. Another approach recently used to discriminate patterns generated from healthy and pathological states as well as aging is based on frequency and rank order statistics of symbolic sequences because complex physiological signals may carry unique dynamic signatures related to their underlying mechanisms [Shieh et al. 2006]. In order to produce a better estimation of the activity, health status or autonomy, heterogeneous data coming from multi-sensor acquisitions might be combined, a procedure called data fusion, using different techniques based on evidence or probabilistic theories [Virone et al. 2006].

\subsection{Limitations of the Reviewed Monitoring Systems}

A common limitation to all systems analyzed in this paper is the small number of subjects participating in implementation or validation phases. This fact makes it hard to extrapolate the results. Another common problem is the reduced number of representative samples [Davis and Fox 2007] or very short series of testing [Shieh et al. 2006; Fogarty et al. 2006]. Some of the studies are even based on simulated data of different behaviors on long or short periods [Virone et al. 2002].

Monitored activities are dependent on the type of sensors used. For example, the microphone-based sensors used by Fogarty et al. [2006] allow detection of what sink is in use and cannot recognize more specific activities at sinks. Other sensors have the potential to capture more activities in a home but the activities captured depend on the places the sensors are installed. While the ability to install a contact switch nearly anywhere in the home, most of the sensors in a study by Tapia et al. [2004] were installed in the kitchen or bathroom, with success in detecting activities such as meal preparation and toileting.

When subjects involved in the study are required to interact with the new technology, new challenges arise and impact the study result. This was the case of the study by Tapia et al. [2004], which required the two participants to use PDAs running software especially designed to label the subject's activities. The participants needed 
to answer to several questions regarding their activities every 15 minutes for 14 days. According to Tapia et al. [2004], subjects had a difficult time adjusting to the experience sampling device and did not enjoy having to tell the computer about doing the same activities repetitively. However, the number of labels was not sufficient for training the machine learning algorithms.

Even if the studies are intended to support independent living, some of the subjects were recruited from assisted living facilities [Chan 2005; Alwan et al. 2006] and others were the researchers and their families [Fogarty et al. 2006] or friends [Shieh et al. 2006]. While this approach provides more data for training the classification algorithm we believe that it does not contribute to the validation of the research questions.

Some articles mentioned as a major limitation low sensitivity of the algorithms, which can be increased by adding more sensors to the existing system [Cuddihy et al. 2007; Alwan et al. 2006], or by careful refinement to reduce the rate of false alerts [Alwan et al. 2006]. The major limitation of all studies is that they assume single persons present in the homes or rooms under investigation. When multiple people are present in a home the number of sensor firings will increase dramatically making possible wrong inferences from the data. Hayes et al. [2004] excluded the data when the subject of their study had visitors.

The majority of studies were experimental or exploratory and some of them even used the Wizard of $\mathrm{Oz}$ technique (use of a human to simulate the response of an unimplemented system). Chen et al. [2007] used both, Wizard of $\mathrm{Oz}$ and experimental study of various sensors and models for detecting and summarizing social interaction among aging parents and caregivers. Rowan and Mynatt [2005] also used Wizard of Oz technique before their pilot study of Digital Family Portrait for enhancing peace of mind of an adult child living 50 miles away of his elderly parent.

\subsection{Recommendations Surfaced from the Presented Systems}

Several recommendations surfaced from the systems presented above. Hayes et al. [2004] identified as an unanswered question in their research how the total activity levels, average walking speed, and patterns of activity through rooms in the home vary across different clinical populations, from those showing normal cognitive processing on standard clinical tests, to those who show minimal cognitive impairment or early cognitive losses. The majority of studies have as a future aim solving the problem of multiple people present in the home instead of ignoring it or even discarding that data [Rowan and Mynatt 2005; Sixsmith and Johnson 2004; Virone et al. 2006].

In order to increase the accuracy of the models used in processing sensor data, some of the authors proposed several solutions of improvement, such as using more powerful techniques like SVM [Eagle and Pentland 2006], a finer granularity of data [Fogarty et al. 2006], fusion of data coming from several sensors [Virone et al. 2002; Virone et al. 2006] or identifying and using an optimal size of data sets [Gil et al. 2007]. Still, in most cases, the correlation between the information produced by the systems and the clinical reality will have to be performed by a physician [Virone et al. 2002 ], and this requires building of interfaces to show this information in ways that 
communicate most intuitively [Gil et al. 2007].

All studies presented in this paper tried to validate their algorithms or research questions by using different approaches and instruments involving the participants, family members or care providers. According to Rowan and Mynatt [2005], in the literature there is an absence of research data on a person's movement in his or her own house that is not biased by self-report or by third party observation and all articles follow this observation. None of them used the approach of trying to connect clinical data of the subjects, which is not biased in any way, to their sensor data. This work attempts to make this connection by trying to find a link between abnormal levels of daily activities (expressed by the levels of motion within the house, restlessness, and possible pulse and breathing) and change in health status (for example, subtle declines in capabilities or behavior caused by elevated pulse pressure) and validate them with real clinical data.

\section{METHODOLOGY}

Among the predictors of functional decline are mentioned activities of daily living which are measured in the medical field by using clinical tools of assessment. Usually, they are based on patients self-reporting or third party observations, as family members or nurses, procedures that bias the results. Even continuous monitoring using wireless sensor technology poses many challenges, and validation process is also based on self-reporting or third party observation. Another predictor of multiple diseases characteristic in elderly people is the pulse pressure. Although pulse pressure is calculated from blood pressure assessment, as the difference between systolic and diastolic BP, it often fades into the background of vital signs. For example, few clinicians would be alarmed by a $\mathrm{BP}$ of $135 / 55 \mathrm{~mm} \mathrm{Hg}$ (that is, a pulse pressure of $80 \mathrm{~mm} \mathrm{Hg}$ ) obtained during a routine visit in a 75-year old patient [Swaminathan and Alexander 2006].

There are biological differences in older people, so that usual symptoms are often not present and there may be a reduction in sensitivity and specificity of any one traditional symptom [Shieh et al. 2006]. The result of these factors is that an older person may show the presence of disease in total body responses and changes in overall levels of functioning. Traditional methods of diagnosis fail to demonstrate this transition, and there is a need for methodologies that will provide objective and continuous assessments of an individual function that can be used for a person in their own home. It is important to obtain information for detecting conditions in their early stages and/or for maintaining health care in daily life. For this purpose it is desirable to measure physiological and vital signs without attaching any biological sensors to the body or making a burden of the measurement [Shieh et al. 2006].

The facts mentioned above shaped the methodology used in this study, which is based on data mining techniques, namely, classification algorithms. The six sensor reading (features) that were investigated in this paper were day and night motion level, day and night restlessness level and day and night heart rate levels, 24 hours prior to the day of measuring the blood pressures. For computing the level of restlessness we employed the sum of the level one firings only. This choice of the 
features is based on the intuition that a normal pattern or daily life supposes high level of activity, i.e. motion, during the day with short or absent periods spent in the bed, while during the night the opposite holds, i.e. long period spent in the bed with low level of restlessness and low level of activity throughout the apartment.

Vital signs of the residents at TigerPlace, including the blood pressures, are not checked daily but more on a need basis or at the regular 6-months check up. Even if the blood pressure would be measured daily, this would not increase the chances of capturing transient abnormalities. Transient abnormalities cannot always be captured during a doctor visit [Yang et al. 2004]. For example, many cardiac diseases are associated with episodic rather than continuous abnormalities such as transient surges in blood pressure, paroxysmal arrhythmias or induced or spontaneous episodes of myocardial ischemia [Yang et al. 2004]. These abnormalities are important but their timing cannot be predicted and much time and effort is wasted in trying to capture an "episode" with controlled monitoring. Important and even-life threatening disorders can go undetected because they occur only infrequently and may never be recorded objectively [Yang et al. 2004]. High risk patients such as those with endstage ischemic heart disease or end-stage myocardial failure often develop life threatening episodes of myocardial ischemia or ventricular arrhythmia. These episodes, if reliably detected would lead to better targeting of potentially life saving [Yang et al. 2004].

The gap in detecting a heart disease in an early stage of development can be filled by using data generated by the in-home monitoring system and processed using data mining techniques. The data mining approach will follow the subsequent steps:

- Data selection

- Data preparation

- Algorithm selection

- Data classification and validation

\subsection{Data Selection}

Sensor data stored on the server are available from any computer with remote access to the server through a web-based visualization interface, currently under refinement. All data available for visualization are grouped by resident and can be displayed for a particular period of time, day by day, for longer intervals, such as weeks or months, or in one, two, up to 12 hour increments for intervals up to two weeks. Several chart options and types are available to the user. Chart options include activities that are monitored by the sensors: motion, restlessness, breathing, pulse, and temperature. Chart types include line, histogram, or pie. The line and histogram charts share the same characteristics: the horizontal axis (X-axis) represents time (days or hours) and the vertical axis ( $\mathrm{Y}$-axis) represents hits of the sensors (number of sensor firings) corresponding to days or hours represented on the $\mathrm{X}$-axis. The pie chart is cumulative and shows the total number of sensors firings corresponding to a particular activity during the desired time period (the time increment is ignored in this case).

A data visualization interface was used to select the data sets that ensure reliable and complete data. The sensor data for 24 hours before each date with blood pressure 
measurements available was visually analyzed and dates with missing data for more than three hours (e.g. the resident was out of the apartment) or uncorrelated sensors firings (e.g. restlessness firings present but no pulse and/or breathing firings during the same hours and vice versa) were not included in the data set. The results of the data selection dictate the size of the data sets and implicitly, the number of subjects because the sensor data is grouped by resident. The larger the data set, the better, and therefore, extremely small data sets were not considered and the corresponding subjects were not included in this study.

\subsection{Data Preparation}

This step implies aggregation of motion and restlessness data by day (7:00 AM to 9:00 PM) and night (9:00 PM to 7:00 AM), 24 hours before each day when blood pressures were measured. For this task a small piece of software was developed which is able to connect to the database, perform the aggregation and write the results in a data file. The content of this file is imported in a spread sheet and the pulse pressure values are added to each resulting row. The data is ready to be further imported in the MATLAB workspace.

\subsection{Algorithm Selection}

For this exploratory study we chose two approaches for predicting the elevated PP events. In the first approach, we used a robust linear regression to estimate the pulse pressure. Then, we calculated the trend of the measured pulse pressure as the average value of the last seven measurements. By continuously computing the pulse pressure and comparing it with the trend of the measured PP, we may alert the nursing staff when some predefined variability limits are exceeded. This approach may provide additional blood pressure monitoring for the elderly persons susceptible to blood pressure variations during the time between two nursing visits.

The independent variables used in the robust regression were the features identified above, i.e. total motion and restlessness per day and night, and the dependent variable was the pulse pressure level. The robust regression is less sensitive to outliers in the data as compared with ordinary least squares regression [Holland and Welsch 1977]. The predicted values of the PP, $\hat{y}_{i}$, were calculated using a robust linear regression as [Hogg 1979]:

$$
\hat{y}_{i}=\sum_{j=1}^{M} x_{i j} \beta_{j}+\beta_{0},
$$

where $x_{i} \in R^{M}$ is the input vector of $M$ sensor measurement, $M \in\{2,3, \ldots, 6\}$, and $\beta \in \mathrm{R}^{\mathrm{M}+1}$ is the vector of regression coefficients. The regression coefficients are computed using an iterative procedure that minimized the criterion:

$$
\mathrm{S}=\sum_{i=1}^{N} \Psi\left(\mathrm{y}_{\mathrm{i}}-\hat{\mathrm{y}}_{\mathrm{i}}\right)
$$

The algorithm used for calculating the PP values and trends has the following steps: 


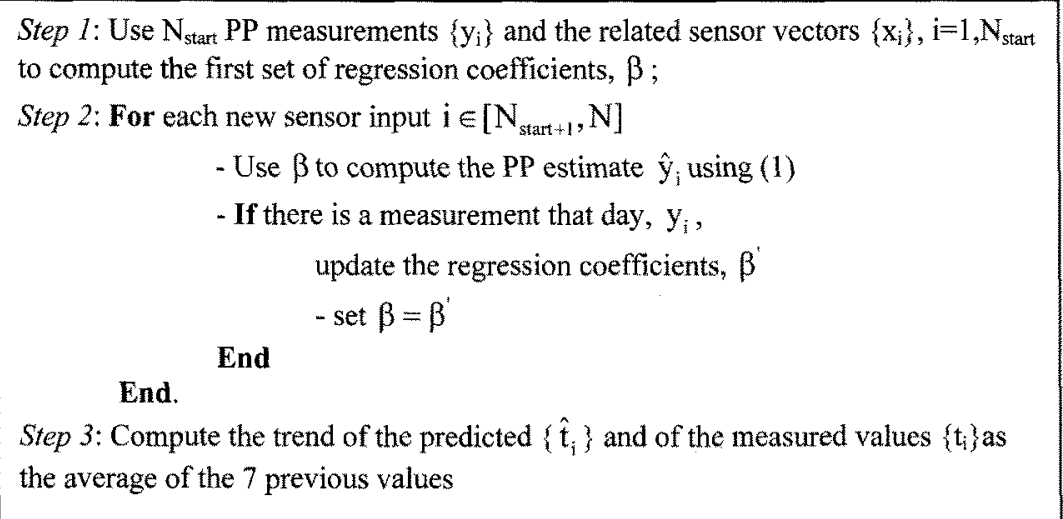

The robust regression is more resilient to outliers by replacing the default least square function $\Psi(t)=t^{2} / 2$ with one that has decreasing weights for outliers. A popular choice for $\Psi$ is Tukey's biweight (bisquare) function [Hogg 1979] $\Psi(t)=t(1-$ $\left.(\mathrm{t} / \mathrm{k})^{2}\right)^{2}$ for $|\mathrm{t}| \leq \mathrm{k}$ and 0 else, where $\mathrm{k}=4.685$ is a tuning constant. In this paper we used a robust regression implementation, robustfit, found in the statistical toolbox of MATALB (http://www.mathworks.com).

In the first approach, the elevated PP events were found using the decision rule: $\hat{y}_{i}>\mu\left(\left\{t_{i}\right\}\right)+c \sigma\left(\left\{t_{i}\right\}\right)$, that is, we declare an alert when the predicted PP is greater than the trend (mean) of the measured PP plus some constant $\mathrm{c}$ multiplied by the standard deviation of the measured PP.

In the second approach, we treated the detection of elevated PP as a two class problem: normal PP $(\mathrm{PP}<60 \mathrm{mmHg})$ and elevated $\mathrm{PP}(\mathrm{PP} \geq 60 \mathrm{mmHg})$. For this task, we used three classification approaches: neural networks (NN), Support Vector Machines (SVM) and a robust linear regression. The training values for the classifiers were 0 (for the normal PP values) and 1 (for the elevated ones). The classification algorithms were also implemented in MATLAB. For the NN approach we used the MATLAB Neural Networks Toolbox, for the SVM we used the MATLAB Bioinformatics Toolbox and for the robust linear regression we used the MATLAB Statistics Toolbox (robustfit). The size of the neural network was M-M-1, meaning that the hidden layer had size $M$, where $M$ was the number of sensor inputs. The challenge that we faced in using NN in this study was due to insufficient data. For example, a 4-4-1 network would require roughly 160 training data samples, which we did not have available in any of our cases. Finally, in this approach, when a prediction of elevated pulse pressure is made, the nursing staff is alerted.

\subsection{Data Classification and Validation}

For the purpose of this study the value of $60 \mathrm{mmHg}$ was used for the abnormal PP threshold. However, further analysis is required for choosing the PP threshold given the controversy existent in literature regarding the abnormal levels of PP and the variation of $\mathrm{PP}$ with age (Figure 1).

In the first approach, the feature selection (the input variables in the model) was 
performed by computing the average relative error (ARE) between the predicted PP trend $\left\{\hat{t}_{\mathrm{i}}\right\}$ and the measured one $\left\{\mathrm{t}_{\mathrm{i}}\right\}$ as:

$$
\operatorname{ARE}=\frac{\sum_{i=1}^{N_{\text {test }}} \frac{\left|t_{i}-\hat{t}_{i}\right|}{t_{i}}}{N_{\text {test }}}[\%],
$$

where $\mathrm{N}_{\text {test }}$ is the number of test (predicted) values.

In the second approach, we used leave-one-out cross-validation in order to evaluate the classification accuracy for each algorithm. ROC curves were used for comparing the classification models. We employed the following algorithm to compute the ROC curves:

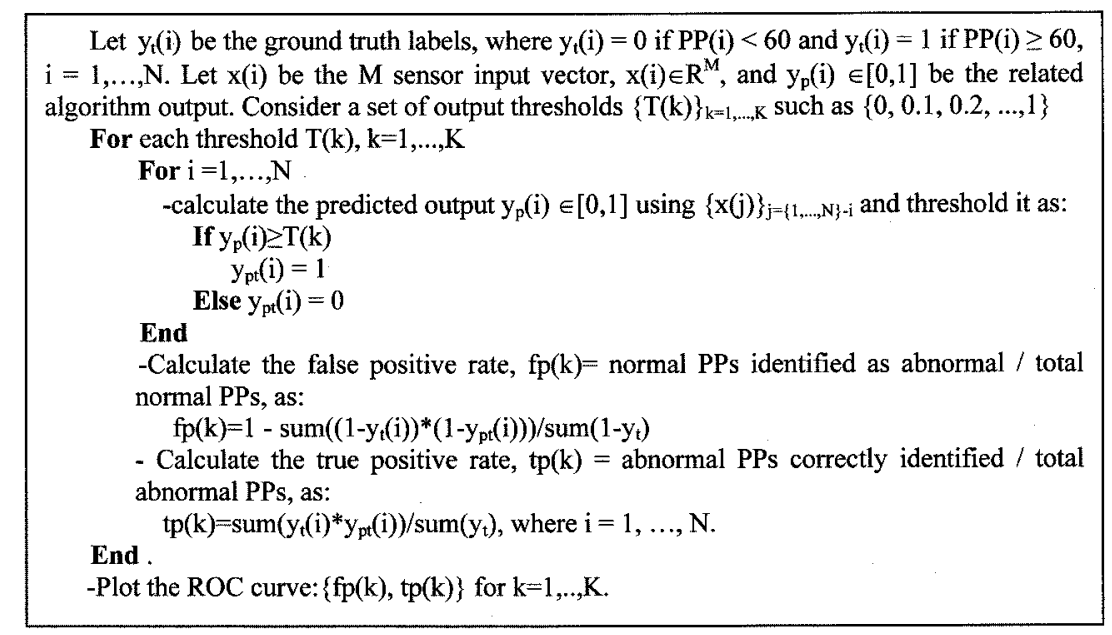

\section{RESULTS}

\subsection{Available Data}

After performing the first step of the data mining approach, only two residents have met the inclusion requirements in this study. The table below summarizes the results of the data selection.

The "out of the room" data was due to the resident being out of his apartment for more than three hours. We are currently working on an algorithm that will reliably detect when the resident is out of his apartment which will increase both the PP prediction accuracy and the amount of available data.

Table I. Results of data selection.

\begin{tabular}{|c|c|c|c|}
\hline & $\begin{array}{c}\text { Total } \\
\text { records }\end{array}$ & $\begin{array}{c}\text { Out of } \\
\text { the room }\end{array}$ & Total data set \\
\hline Male & 93 & 52 & $41(30 \mathrm{PP} \geq 60)$ \\
\hline Female & 139 & 49 & $90(35 \mathrm{PP} \geq 60)$ \\
\hline
\end{tabular}


Table II. The average relative error (ARE) for the PP trend for the two residents in the study.

\begin{tabular}{|c|l|c|c|}
\hline No. & Sensor type & ARE for Male [\%] & ARE for Female [\%] \\
\hline 1 & motion & 3.1 & 8.5 \\
\hline 2 & motion +restlessness & 3.8 & 9.8 \\
\hline 3 & motion + restlessness + heart rate & 10.5 & 11.5 \\
\hline 4 & motion + heart rate & 7.4 & 10.7 \\
\hline 5 & Restlessness + heart rate & 4.2 & 15.5 \\
\hline 6 & day motion + day restlessness & 3.7 & 9.2 \\
\hline
\end{tabular}

\subsection{The Results of the First Approach: using the PP Trend to Predict the Abnormal Clinical Events}

We have tested various combinations of the three available sensors (motion, heart rate and restlessness) during day and/or night time. The average relative errors for the tested combinations for the two available residents (Male and Female) are given in Table II.

The average relative errors from Table II are mostly below $10 \%$ which denotes that the PP trend can be predicted reasonably well. The day and night total motion (2 variables, row 1) seem to predict best the PP trend in both cases. The resulting PP plots for this case are shown in Figure 2 and 3.

By inspecting the figures 2 and 3 we see that there are regions (circled) where the predicted PP trend differ significantly from the measured trend. Our hypothesis is that during those periods of time the subjects had experienced settled changes caused maybe by some other clinical events not related to blood pressure, idea that was partially confirmed by the medical records. The unconfirmed discrepancies might be due to the tendency of the elderly of not reporting what they consider

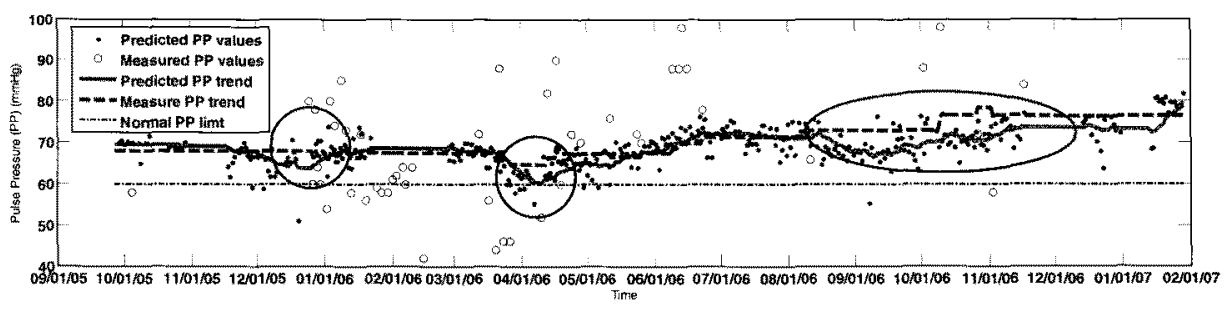

Figure 2. The predicted and measured pulse pressure for the Male resident.

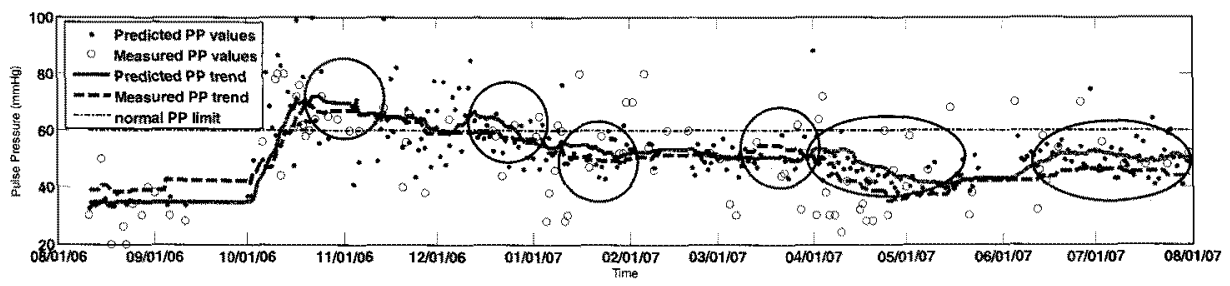

Figure 3. The predicted and measured pulse pressure for the Female resident. 

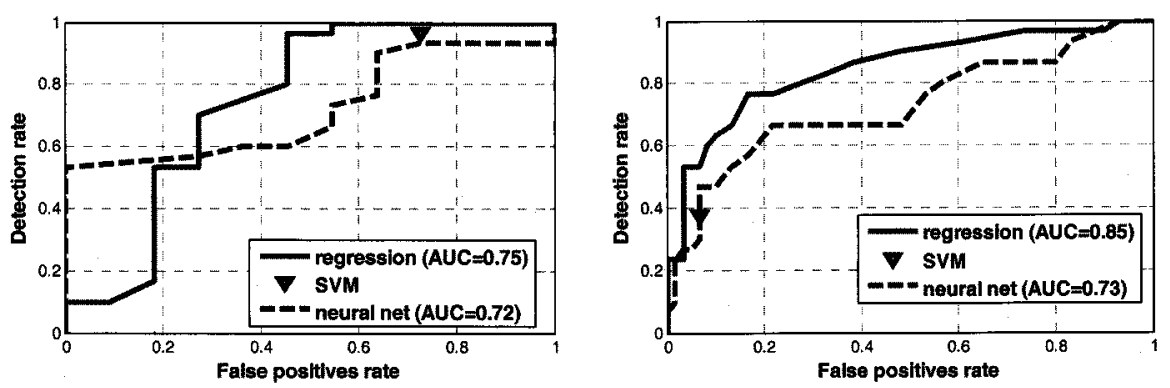

Figure 4. ROC curves for Male(left) and Female(right) with $M=4$ and 3 algorithms.

minor events. We especially observed this fact in our Male resident who, for the last part of 2006, described his nights in his health journal as "same old night". It turned out that, in fact, his health was deteriorating and he passed away several months later.

Using the threshold of one standard deviation $(c=1)$ from the measured PP trend we predicted 5 of the 33 measured elevated PP events for the female resident with 17 false alarms, while none of the 26 incidents were predicted for the male resident and no false alarms. By lowering the threshold to $0.5 \sigma$ we predicted 11 of the 33 elevated $\mathrm{PP}$ events with 60 false alarms for the female and 2 out of 26 with 3 false alarms for the male resident, respectively. The false alarms include predicted elevated PP events that might have been right but no blood pressure measurement was taken because the resident did not complain that he was not feeling well. While this approach does not seem to predict well the elevated PP episodes, we believe that the trend comparison might be used for detecting unreported periods of time when the resident does not "feel well".

\subsection{The Results of the Second Approach: using Classifiers to Predict Abnormal PP}

In the second approach, we used three classifiers: SVM, robust linear regression and neural nets to predict abnormal PP events. Here, we used only the measured PP samples trying to predict if the measurement is elevated or not. A false alarm here signifies that we predicted a measurement to be elevated when it was not. In this experiment we used four features $(M=4)$ : night motion, day motion, night restlessness and day restlessness. For SVM, we did not compute the ROC curve (we show just one pair \{true positive rate, false positive rate\}). A comparison of the three algorithms is shown in Figure 4.

The above ROC curves show an average AUC of about 0.8 . This result gives us a reason to further investigate this method.

\section{CONCLUSIONS AND FUTURE WORK}

The main purpose of this project was to find a link between the sensor data generated by the in-home monitoring system in place at TigerPlace and vital signs, i.e. pulse pressure, of participants connected to the sensor network. Different classification 
algorithms including neural network, robust linear regression, and SVM have been applied to two data sets corresponding to a male and female living at TigerPlace. The results suggest that the bed restlessness and motion levels may be used to predict elevated pulse pressure in elderly between two nursing appointments.

However, more data and more subjects are necessary in order to support the results of any of the previous methods. We are currently taking daily measurement of the blood pressure of several residents connected to the in-home monitoring system. We hope to accumulate several months of daily sensor data and blood pressure measurements together with all the significant clinical events. In addition, a result fusion (ex. using a 2-out-of 3 voting scheme) might substantially reduce the false alarm rate. Also, other machine learning algorithms such as AdaBoost can be used in conjunction with the SVM and neural network algorithms to improve their performances.

Other improvements are related to factors that influence the sensor readings such as the presence of visitors, the time out of the apartment and the sleep duration that we only partially accounted for. We are currently working on algorithms to detect and correctly integrate the above factors.

\section{REFERENCES}

Alwan, M., Dalal, S., Mack, D., Kell, S., Turner, B., Leachtenauer, et al. 2006. Impact of monitoring technology in assisted living: outcome pilot. IEEE Transaction on Information Technology in BioMedicine, 10(1), 192-198.

Atrinson, H. H., Cesari, M., Kritchevsky, S. B., Penninx, B. W. J. H., Fried, L. P., Guralnik, J. M., et al. 2005. Predictors of combined cognitive and physical decline. Journal of the American Geriatrics Society, 53(7), 1197-1202.

Blacher, J., Staessen, J. A., Girerd, X., Gasowski, J., Tujus, L., Liv, L., et al. 2000. Pulse pressure not mean pressure determines cardiovascular risk in older hypertensive patients. Archives of Internal Medicine, 160(8), 1085-1089.

Chan, M., CAmpo, E., Esteve, D. 2005. Assesment of activity of elderly people using a home monitoring system. International Journal of Rehabilitation Research 28(1), 69-76.

Chen, D., Yang, J., Malkin, R., Wactlar, H. D. 2007. Detecting social interactions of the elderly in a nursing home environment. ACM Transactions on Multimedia Computing, Communications and Applications, 3(1), 1-22.

Cuddihy, P., Weisenberg, J., Graichen, C., And GANesh, M. 2007. Algorithm to automatically detect abnormally long periods of inactivity in a home. In Proceedings of the 1 st ACM SIGMOBILE international Workshop on Systems and Networking Support for Healthcare and Assisted Living Environments. New York: ACM Press. 89-94.

DAVIS, M. G., FOX, K. R. 2007. Physical activity patterns assessed by accelerometry in older people. European Journal of Applied Physiology, 100(5), 581-589.

Eagle, N., Pentland, A. 2006. Reality mining: sensing complex social systems. Personal and Ubiquitous Computing, 10(4), 255-268.

FogArTY, J., Au, C., HudSON, S. E. 2006. Sensing from the basement: a feasibility study of unobtrusive and low-cost home activity recognition. In Proceedings of the 19th Annual ACM Symposium on User interface Software and Technology. New York: ACM Press. 91100.

Franikln, S. S., Larson, M. G., Khan, S. A., Wong, N. D., Leip, E. P., Kannel, W. B., et al. 2001. Does the relation of blood pressure to coronary heart disease risk change with aging? The Framingham heart study. Circulation, 103(9), 1245-1249.

Gil, N. M., Hine, N. A., Arnott, J. L., Hanson, J., Curry, R. G., Amaral, T., et al. 2007. Data visualization and data mining technology for supporting care for older people. 
Proceedings of the $9^{\text {th }}$ International ACM SIGACCESS Conference on Computers and Accessibility. New York: ACM Press. 139-146.

Glynn, R. J., Chae, C. U., Guralnik, J. M., Taylor, J. O., Hennekens, C. H. 2000. Pulse pressure and mortality in older people. Archives of Internal Medicine, 160(18), 2765-2772.

HAN, J., KAMBER, M. 2006. Data mining: concepts and techniques. San Francisco: Morgan Kaufmann Publishers.

Hayes, T. L., PAvel, M., KAyE, J. A. 2004. An unobtrusive in-home monitoring system for detection of key motor changes preceding cognitive decline. Proceedings of the $26^{\text {th }}$ Annual International Conference of the IEEE EMBS. San Francisco. 2480-2483.

Holland, P. W., Welsch, R. E. 1977. Robust regression using iteratively reweighted least squares. Communications in Statistics Theory and Methods, 6(9), 813-827.

LEE, M. L. T., Rosner, B. A., WEISS, S. T. 1999. Relationship of blood pressure to cardiovascular death: the effects of pulse pressure in the elderly. Annals of Epidemiology, 9(22), 101-107.

Mitchell, G. F., Vasan, R. S., Keyes, M. J., Parise, H., Wang, T. J., LARSon, M. G., et al. 2007. Pulse pressure and risk of new-onset atrial fibrillation. Journal of the American Medical Association, 297(7), 709-715.

Otto, C. A., Jovanov, E., Milenkovic, A. 2006. A WBAN-based system for health monitoring at home. Proceedings of the 3rd IEEE-EMBS International Summer School and Symposium on Medical Devices and Biosensors MIT. Boston. 20-23.

Peters, R., Marero, C. M., Pinto, E., Beckett, N. 2007. Hypertension in the very elderly. Aging Health, 3(4), 517-525.

Rantz, M. J., MareK, K. D., Aud Ma, Johnson Ra, Otto D., Porter D. 2005. TigerPlace: A New Future for Older Adults. J. of Nursing Care Quality, 20(1), 1-4.

Romero, N., Markopoulos, P., Baren, J., Ruyter, B., IJsselsteiJN, W., Farschchian, B. 2007. Connecting the family with awareness systems. Personal and Ubiquitous Computing, 11(4), 299-312.

Rowan, J., MYNATT, E. D. 2005. Digital Family Portrait field trial: support for Aging in Place. In Proceedings of the SIGCHI Conference on Human Factors in Computing Systems. New York: ACM Press. 521-530.

Ruyter, B., Pelgrim, E. 2007. Ambient assisted-living research in CareLab. Interactions, 14(4), 30-33.

Safar, M. E., LAJemi, M., Rudnichi, A., Asmar, R., Benetos, A. 2004. Angiotensin-converting enzyme $\mathrm{D} / \mathrm{I}$ gene polymorphism and age-realted changes in pulse pressure in subjects with hypertension. Arteriosclerosis, Thrombosis, and Vascular Biology, 24(4), 782-786.

Schultz-Larsen, K., Avlund, K. 2007. Tiredness in daily activities: a subjective measure for the identification of frailty among non-disabled community-living older adults. Archives of Gerontology and Geriatrics, 44, 83-93.

Shieh, J. S., Chuang, C. S., Wang, X., Kuo, P. Y. 2006. Remote monitoring of mobility changes of the elderly at home using frequency rank order statistics. Journal of Medical and Biological Engineering, 26(2), 81-88.

Sixsmith, A., JoHnson, N. 2004. A smart sensor to detect the falls of the elderly. IEEE Pervasive Computing, 3(2), 42-47.

SwaminathaN, R. V., AleXANDeR, K. P. 2006. Pulse pressure and vascular risk in the elderly: associations and clinical implications. The American Journal of Geriatric Cardiology, 15(4), 226-232.

TAPIA, E. M., InTILle, S. S., LARSON, K. 2004. Activity recognition in the home using simple and ubiquitous sensors. Proceedings of the Second International Conference on Pervasive Computing. Springer. 158-175.

Teaw, E., Hou, G., Gouzman, M., Tang, K. W., Kesluk, A., Kane, M., et al. 2005. A wireless health monitoring system. Proceedings of the 2005 IEEE InternationalConference on Information Acquisition. 247-252.

Virone, G., NourY, N., DEMONGEOT, J. 2002. A system for automatic measurement of circadian 
activity deviations in telemedicine. IEEE Transactions on Biomedical Engineering, 49(12), 1463-1469.

Virone, G., Wood, A., Selavo, L., Cao, Q., Fang, L., Doan, T., et al. 2006. An assisted living oriented information system based on a residential wireless sensor network. Proceedings of the $1^{\text {st }}$ Distributed Diagnosis and Home Healthcare (D2H2) Conference. Arlington, VA. 95-100.

Williams, A., Ganesan, D., Hanson, A. 2007. Aging in place: fall detection and localization in a distributed smart camera network. Proceedings of the $15^{\text {th }}$ International Conference on Multimedia. New York: ACM Press. 892-901.

YAnG, G. Z., Lo, B., Wang, J., Rans, M., ThiemJarus, S., NG, J. 2004. From sensor networks to behavior profiling: a homecare perspective of intelligent buildings. Proceeding of the IEE Seminar for Intelligent Buildings, 1-7.

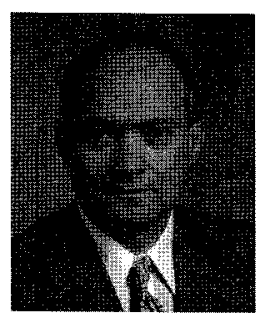

Mihail Popescu received a Master of Science in Medical Physics in 1995, a Master of Science in Electrical Engineering in 1997 and a PhD in Computer Science in 2003 from the University of Missouri, Columbia, Missouri. He is currently an assistant professor in the Department of Health Management and Informatics at the University of Missouri at Columbia. His research interests include eldercare technologies, fuzzy logic and ontological data mining. Dr. Popescu is a senior IEEE member.

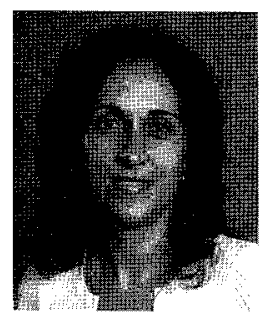

Elena Florea received the Bachelor of Science degree in Mechanical Engineering from the Transylvania University Brasov. She is the recipient of a Master of Science in Industrial Engineering from the University of Missouri, Columbia and currently is a candidate for a Master's of Science in Health Informatics from the same university. Her research interests include database, data mining, and unobtrusive sensing technologies. Current research focuses on the application of wireless sensor data to continuous health monitoring of elderly people. 